\title{
Simulating Propellant Reorientation of Vehicle Upper Stage in Microgravity Environment
}

\author{
Zhang-Guo Li • Zhi-Qiang Zhu • Qiu-Sheng Liu • \\ Hai Lin · Jing-Chang Xie
}

Received: 15 December 2012 / Accepted: 5 September 2013 / Published online: 28 September 2013

(C) Springer Science+Business Media Dordrecht 2013

\begin{abstract}
The computational and experimental studies have been performed to investigate the hydrodynamic process of liquid propellant reorientation for the launch vehicle series fuel tanks in microgravity environment. The VOF method was used to simulate the free surface flow of gas-liquid. The process of the liquid propellant reorientation started from initially curved interface at low Bond number. The propellant reorientation flow procedure at high Bond number was obtained from numerical simulation and scale model experiment in drop tower. The numerical results agreed well with the experiments. The results can be used to adjust the engineering reorientation parameters.
\end{abstract}

Keywords Propellant management · Launch vehicle . Reorientation · Drop tower

\section{Introduction}

The main engine of launch vehicle must have the ability to restart many times in microgravity environment in order to fit different transmission requirements such as deep space exploration, During the engine-off time, the liquid propellant is expected to be held in the proper position of the tank for an engine restart on the vehicle. In space, where the effective gravity is small, that the effects of residual gravity and capillary pressure determine the free surface location.

Z.-G. Li · Z.-Q. Zhu · Q.-S. Liu $(\bowtie) \cdot$ H. Lin · J.-C. Xie

Key Laboratory of Microgravity, Institute of Mechanics,

Chinese Academy of Sciences, Beijing 100190, China

e-mail: liu@imech.ac.cn

Z.-G. Li

e-mail: lizhangguo@imech.ac.cn
Capillary pressure is computed by surface tension times curvature including the contact angle as a boundary condition (Dodge 1990). Spacecraft must therefore complement methods to ensure that the liquid is located at the desired location in the tank even when effective gravity is directed adversely or in unknown directions. Similarly, if the tank is pressurized and must be vented periodically, methods are required to ensure that gas is positioned over the vent. The most dangerous case of propellant reorientation is that the liquid phase locates at the opposite direction. As Fig. 1 shown, assuming the liquid fuel outlet at the bottom of the tank, Here the black area denotes liquid, the gray denotes gas. A useful way to accomplish this objectives is to use an auxiliary thrusting system to provide a linear acceleration large enough to settle the liquid (Hochstein et al. 1991; Hochstein and Chato 1992). Its principles are similar to a tank on Earth exposed to gravity. In general, the thrusters in typical upperstage vehicle are used to settle the propellant in the tank. So, the thrust and time required to reorient the liquid propellant back to its desired location must be known to ensure a successful engine restart sequence and to determine effective thruster parameters (Table 1). Theoretical analysis of liquid behavior in microgravity (Stark et al. 1974; Dodge 1971) indicate that the initial state of the gas-liquid interface will form a curved surface at low Bond number under micro-gravity condition. After the acting of auxiliary thrusting system, the propellant reorientation flow in the tanks with a quite high Bond number $(B o>50)$.

The reorientation flow was dominated by volume force of the thrust with the characteristic time scale

$T c=\sqrt{R / g}$

In the reorieatation process, the sloshing liquid wrapped into large bubbles in the tank. And gegeyser generated, finally 


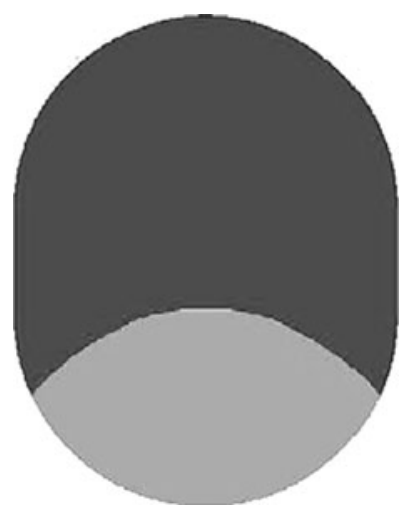

Fig. 1 Free surface configuration in propellant tanks under microgravity environment

bubbles broke up and discharged from the liquid. Baumbach et al., (Hopfinger and Baumbach 2008) presented the liquid sloshing in partially filled circular, cylindrical tanks by a sudden change in Bond number and analyzed the transient behavior of a large bubble in a vertical tube of finite length (Baumbach et al. 2005). In our research, the real scale tanks of vehicle upper stage are in large size, we can't take the real size model experiment in drop tower platform. So the scale model experiments with similarity law in Bond number and Froude number were carried out.

\section{Drop Tower Experiments}

The experimental investigation was conducted in the Drop Tower Facility of National Microgravity Laboratory of China (NMLC) with scale models of liquid propellant tanks. The drop capsule was released in the drop tower to yield variable reduced gravity conditions (from $10^{-3} g_{0}$ to $0.146 g_{0}, g_{0}=9.81 \mathrm{~m} / \mathrm{s}^{2}$ )for an experiment time up to 3.6 seconds. The experimental setup consisted of the scale models of tanks, precisely controllable thrusting system, high speed digital recording system, and background illumination device. The reorientation process was recorded by a high speed digital recording system. The camera with a resolution of $1024 \times 768$ pixels were used to record the liquid motion with a frame rate of 117 frames/s.

During the reorientation process, the initial conditions of the gas-liquid interface will determine the flow mode of liquid in fuel tanks ( $\mathrm{Li}$ et al. 2009), the reorientation process begins with a curve interface of gas-liquid. So we divided

Table 1 Definition of the dimensionless numbers. $\Delta \rho$ is the difference in the density of the liquid and the gas, $\sigma$ the surface tension, $\mathrm{R}$ the radius of the tank, $U$ the characteristic velocity

\begin{tabular}{ll}
\hline$B o=\frac{\triangle \rho g R^{2}}{\sigma}$ & Bond number \\
$F r=\frac{U^{2}}{g R}$ & Froude number \\
\hline
\end{tabular}

the experiment in two periods. First, the drop capsule was released in the form by free fall, and the gas-liquid interface changed from flat to curve interface. Then the reorientation thrust started immediately after the interface reach the most similar shape of the space microgravity status. This was the second period, reorientation period. We defined the initial curved interface formed clock as $T_{0}$ for reorientation time origin. The characteristic time of the reorientation process was started at the moment of $T_{0}$.

In the series of experiment test, the model tanks used had radius $R$ of 35 and 55 millimeters and length $\mathrm{L} 1$ of 90.4 and 142.0 millimeters, respectively. The experimental fluid FC72 was used in this investigation, as shown in Table 2. The experimental tank is made of plexiglass in order to facilitate observation. FC-72 is a quite well wetting liquid. we take it as the experimental medium because the vehicle tank is made of titanium alloy. And the liquid propellant behave also very wetting to the tank wall.

In the studied cases, we chose the reorientation Bond number conditions of $51.8,55.9,89.5,96.7$ with 35 millimeters tank model 388.2, 419.1, 671.5, 724.8 with 55 millimeters tank corresponding to the thrust value which were available in the vehicle upper stage. A series of video images, recorded with the high-speed digital video system, shows the development of the fluid interface after free fall in the first period of the experiment (Fig. 2) and the reorientation flow after thrust being active in the second period of the experiment (Fig. 3). In this case, the tank had radius of 35 millimeters, and thrust Bond number was 96.7. As described above, the main interest of this study is the investigation of the flow characteristics in the second period of the experiment. As Fig. 3 shown, a series event times from initiation of thrust were defined as below for the reorientation process.

$T_{1}$ : time of liquid impact at tank top, geyser initiate $\left(2^{\text {nd }}\right.$ in Fig. 3);

$T_{2}$ : time of geyser impact on liquid-vapor interface $\left(4^{\text {th }}\right.$ in Fig. 3);

$T_{3}$ : time of geyser impact on tank bottom ( $5^{\text {th }}$ in Fig. 3);

$T_{4}$ : time when tank top out of bubbles ( $6^{\text {th }}$ in Fig. 3);

$T_{5}$ : time when bubbles almost clear from the liquid, reorientation finish.

Sixteen reorientation experiments were conducted (8 cases, each case was repeated once) and the event time were obtained by measurement. Due to the time limit of drop tower experiment (up to 3.6 seconds), $T_{5}$ was not obtained from this experiment. The dimensionless reorientation time which equals $T / T_{c}$ as shown in Table 3.

Table 2 FC-72 properties at $20^{\circ} \mathrm{C}$

\begin{tabular}{lllll}
\hline Liquid & $\sigma, \mathrm{N} / \mathrm{m}$ & $\rho, \mathrm{kg} / \mathrm{m}^{3}$ & $\mu, \mathrm{kg} / \mathrm{m} \cdot \mathrm{s}$ & $\theta,^{\circ}$ \\
FC-72 & $1.0 \times 10^{-2}$ & 1680.0 & $6.4 \times 10^{-4}$ & 10.0 \\
\hline
\end{tabular}



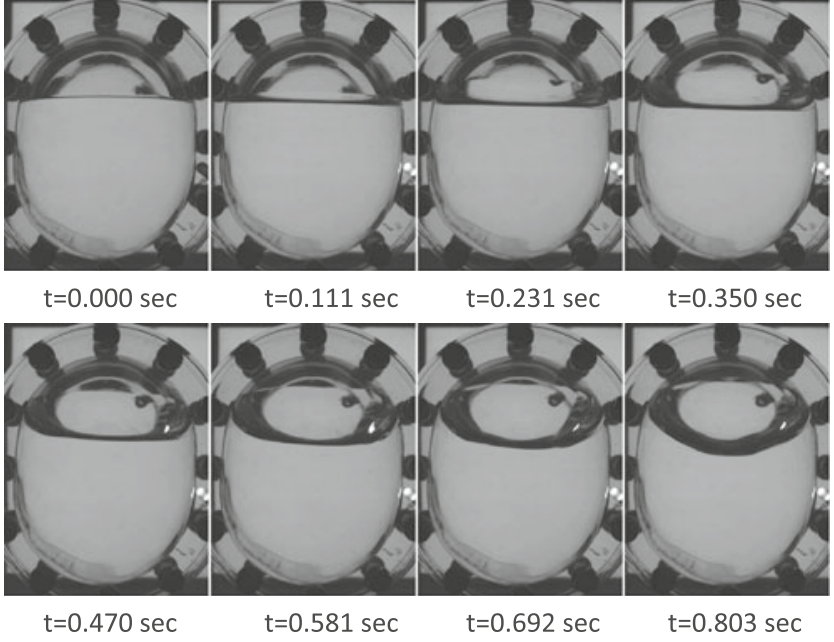

$\mathrm{T}_{0}$

Fig. 2 Development of the free liquid interface in the first period of the experiment (free fall period): $R=35 \mathrm{~mm}$

\section{Numerical Simulation and Discussion}

To simulate the deforming free surface flow the commercial CFD solver Fluent v6.3.26 was used. In this paper, an axisymmetric model is adopted. the volume-of-fluid (VOF) method (Hirt and Nichols 1981; Scardovelli and Zaleski 1999)was used to simulate the free surface flow of gas-liquid. For incompressible flows with constant properties, the continuity, momentum and VOF equations are given by:

$\nabla \cdot \mathbf{v}=0$
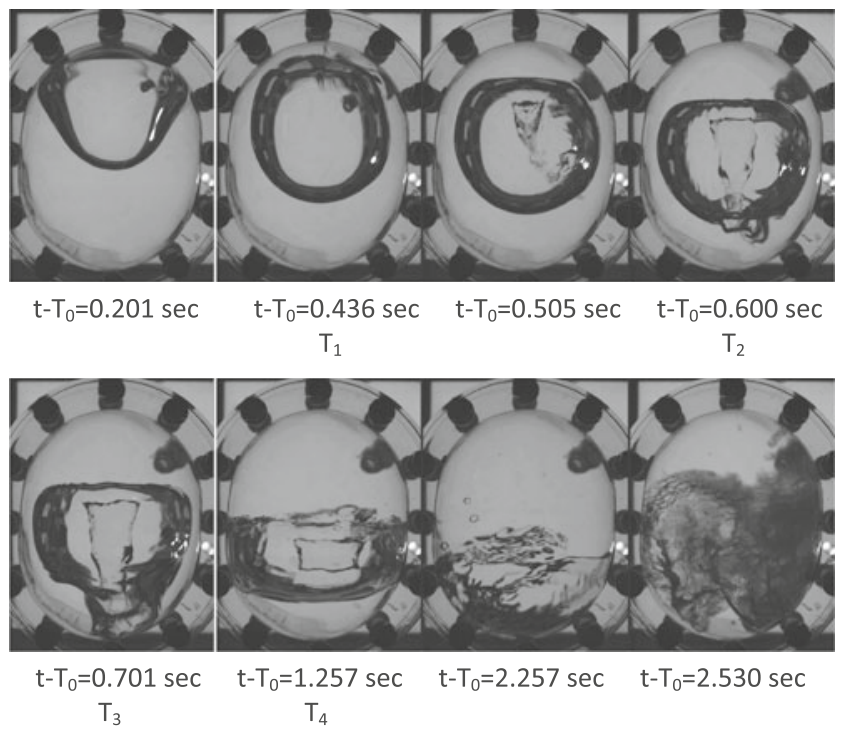

Fig. 3 Development of the free liquid interface in the second period of the experiment (reorientation period): $R=35 \mathrm{~mm}, B o=96.7$
Table 3 Reorientation dimensionless event times of drop tower reorientation experiments

\begin{tabular}{lllllll}
\hline$R, \mathrm{~mm}$ & \multicolumn{1}{l}{ Bo } & $T_{1} / T_{C}$ & $T_{2} / T_{c}$ & $T_{3} / T_{c}$ & $T_{4} / T_{c}$ & $T_{5} / T_{c}$ \\
\hline 35 & 51.8 & 1.31 & 1.74 & 4.58 & - & - \\
35 & 55.9 & 1.61 & 2.14 & 3.18 & - & - \\
35 & 89.5 & 1.02 & 1.34 & 2.75 & 3.63 & - \\
35 & 96.7 & 1.39 & 1.65 & 2.34 & 4.49 & - \\
55 & 388.2 & 1.23 & 1.45 & 2.39 & 3.73 & - \\
55 & 419.1 & 1.47 & 1.66 & 2.52 & - & - \\
55 & 671.5 & 1.62 & 3.37 & 4.66 & 5.69 & - \\
55 & 724.8 & 2.08 & 3.77 & 4.33 & - & - \\
\hline
\end{tabular}

$\frac{\partial \mathbf{v}}{\partial t}+(\mathbf{v} \cdot \nabla) \mathbf{v}=\frac{1}{\rho}\left(-\nabla p+\mu \nabla^{2} \mathbf{v}+\mathbf{f}_{\mathbf{s}}\right)+\mathbf{g}$

$\frac{\partial F}{\partial t}+\mathbf{v} \cdot \nabla F=0$

The symbol $\mathbf{v}$ is the velocity vector; $p, \rho$, and $\mu$ represent the pressure, density, and viscosity of the mixture fluid, respectively. $F$ is the VOF function. The term $\mathbf{g}$ denotes the residual acceleration of the aerodynamic drag or the reorientation thrust acting on the spacecraft for the orbital maneuver course; $\mathbf{f}_{\mathbf{s}}$ is the surface tension at the liquid-gas interface. Surface tension effect plays a significant role in the free surface flow problems in micro-gravity environment. The continuum surface force (CSF) model (Brackbill et al. 1992) was used to model surface tension. In the CSF model, surface tension effect is treated as a body force $\mathbf{f}_{\mathbf{s}}$, It is distributed within a transition region of finite thickness at the interface, given by:

$\mathbf{f}_{\mathbf{s}}=\sigma \kappa \mathbf{n} \delta(\mathbf{x})$

where $\sigma$ is the coefficient of surface tension, $\kappa$ the mean curvature, $\mathbf{n}$ the normal to the surface, and $\delta(\mathbf{x})$ a delta function concentrated at the interface. In the context of the VOF method, the body force is given by:

$\mathbf{f}_{\mathbf{s}}=\sigma \kappa \nabla F$

It is included in the momentum equation as a source term. This continuum treatment of the discontinuous change at the interface eases the implementation of the surface tension effect where only the VOF function is needed. In problems with complex topological changes, the CSF model is superior to the conventional method in robustness and versatility. The term $\sigma \kappa \nabla F$ indicates that $\mathbf{f}_{\mathbf{s}}$ is proportional to the curvature $\kappa$ with the force acting along the normal direction of the interface. Contact angles are applied as a boundary condition at the contact line (Bussmann et al. 1999). In the simulation, we set the contact angle $\theta_{c}=10$. Boundary conditions for fluid along solid surfaces are the no-slip and no-penetration conditions. 


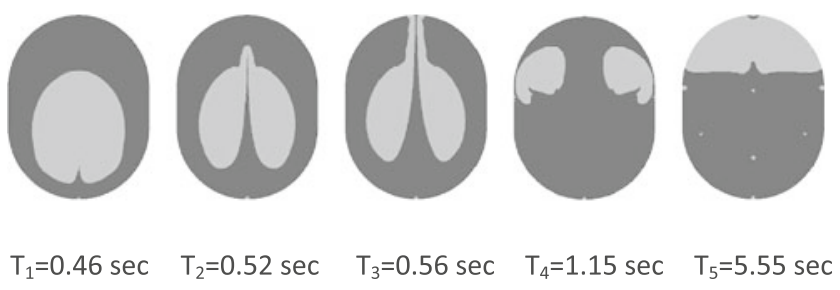

Fig. 4 Numerical simulation of reorientation process at $B o=96.7$ in experiment model tank

For the following simulations, the body force weighted scheme is utilized to compute the face pressure by assuming that the normal gradient of the difference between pressure and body forces is constant. The pressure-implicit with splitting of operators (PISO) (Issa 1986), pressurevelocity coupling scheme is used to obtain a semi-implicit pressure correction equation. The pressure-correction equation is subsequently solved using the algebraic multigrid (AMG) method (Hutchinson and Raithby 1986). Temporal discretization is accomplished using implicit time integration, which is unconditionally stable with respect to time step size. Solutions are subsequently iterated at each time level until the convergence criteria are met (Marchetta and Roos 2009). The geometric reconstruction scheme is used to reconstruct the interface between fluids using a piecewiselinear approach.

According to the on-orbit vehicle situation, the liquid was stabilized at the top of the tank with a curved interface as Fig. 1 shown. The engine would restart in a number of times with different propellant residual, and in each case corresponding to the respective different filling ratio in different fuel tanks.

We choose a typical situation that reorientation Bond number of 96.7, the specific reorientation flow process of experimental scale and real tank scale were shown in Fig. 4 and 5, respectively. Initially, the propellant gas-liquid interface had a concave curvature. During the reorientation progress, the liquid propellant shifted down along the tank wall by the application of thrust. Then the geysering occurred when it impacted the tank bottom. The geyser kept moving due to inertia and geyser tip impacted on the interface then reached the top. In these periods, the single gas-liquid interface broke up, gas liquid mixed and bubbles

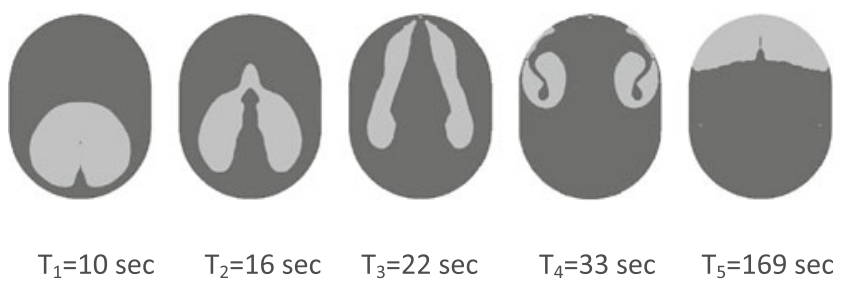

Fig. 5 Numerical simulation of reorientation process at $B o=96.7$ in real scale tank

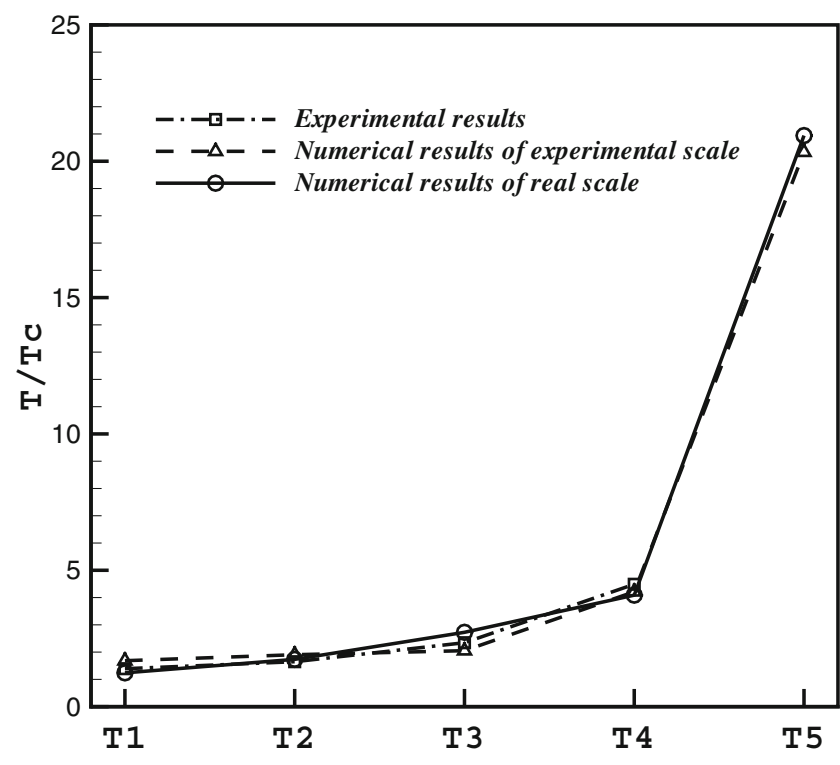

Fig. 6 Reorientation event-time versus time scale at $B o=96.7$

generated from the liquid inside. After the liquid sloshing and bubbles rising, tank top could be clear of liquid. Finally, all the bubbles discharged from the liquid by the settling thrust, the propellant reorientation flow process finished. In the other settling thrusts which were available by the vehicle engine and with different liquid fill ratio, the flow processes were similar. Figures 3, 4 and 5 present the propellant reorientation flow state at each event-time. The results show that the flow modes and the gas-liquid distribution at each eventtime of simulation results agreed well with the experimental results. and the flow modes of scale model results agreed well with the real scale results.

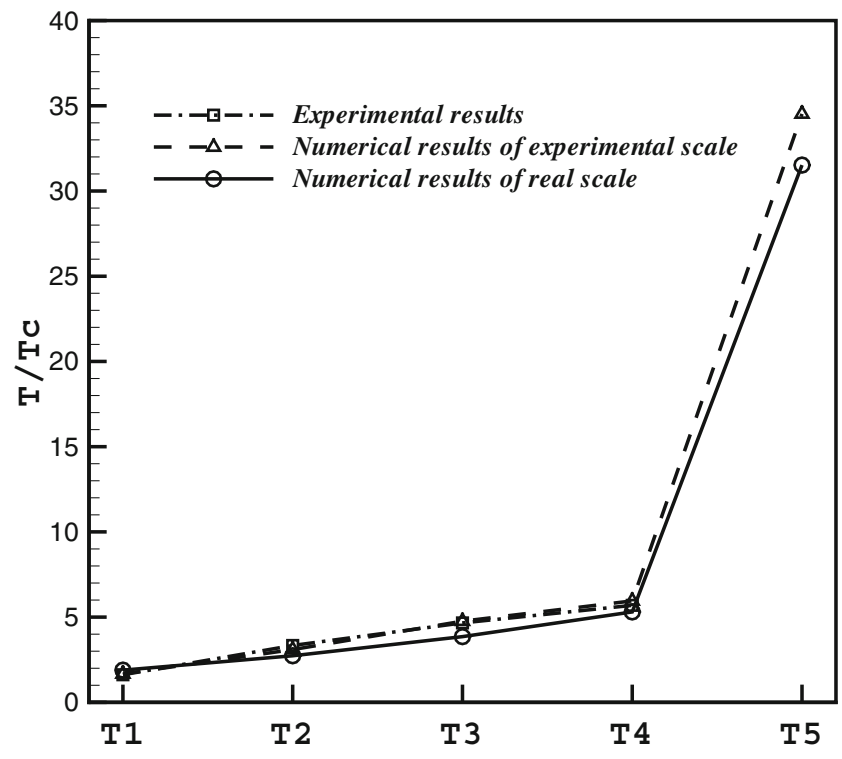

Fig. 7 Reorientation event-time versus time scale at $B o=671.5$ 
The event-time in various numerical simulations were extracted to compare with the experimental results. Figures 6 and 7 show that the dimensionless event-time agreed well in numerical and experimental results.

\section{Conclusion}

Experiments and numerical calculations were performed to investigate the process of liquid propellant reorientation flow dynamics in microgravity environment. The reorientation event-time of propellant reorientation flow was obtained from numerical simulation agreed well with the experimental results. It is a good complement to our previous work on the analysis of reorientation Bond number versus reorientation time and thrust consumption. The reorientation plan proposed in the previous work was validated by this research, and the results can be used to adjust the engineering reorientation parameters.

Acknowledgments This research was financially supported by the National Natural Science Foundation of China (Grants No. 11072249, No. 50890182 and No. 11102211) and the Knowledge Innovation Project of Chinese Academy of Sciences (KGCX-SW-409).

\section{References}

Baumbach, V., Hopfinger, E.J., Cartellier, A.: The transient behavior of a large bubble in a vertical tube. J. Fluid Mech. 524, 131-142 (2005)
Brackbill, J.U., Kothe, D.B., Zemach, C.: A continuum method for modeling surface tension. J. Comput. Phys. 100(2), 335-354 (1992)

Bussmann, M., Mostaghimi, J., Chandra, S.: On a three dimensional volume tracking model of droplet impact. J. Phys. Fluids 11(6), 1406-1417 (1999)

Dodge, F.T.: Low-gravity fluid dynamics and transport phenomena. Aeronaut. Astronaut. 130, 3-14 (1990)

Dodge, F.T.: Further Studies of Propellant Sloshing under Low Gravity Conditions (1971). NASA-CR-119892

Hirt, C.W., Nichols, B.D.: Volume of fluid (VOF) method for the dynanlics of free boundaries. J. Comput. Phys. 39, 201-225 (1981)

Hochstein, J.I., Chato, D.J.: Pulsed thrust propellant reorientation: concept and modeling. J. Propuls. Power 8(4), 770-777 (1992)

Hochstein, J.I., Patag, A.E., Chato, D.J.: Modeling of impulsive propellant reorientation. J. Propuls. Power 7(6), 938-945 (1991)

Hopfinger, E.J., Baumbach, V.: Liquid Sloshing in Cylindrical Fuel Tanks, 2nd European Conference for Aerospace Sciences (EUCASS), pp. 1-8 (2008)

Hutchinson, B.R., Raithby, G.D.: A multigrid method based on the additive correction strategy. Numer. Heat Transf. 9, 511-537 (1986)

Issa, R.I.: Solution of implicitly discretized fluid flow equations by operator splitting. J. Comput. Phys. 62, 40-65 (1986)

Li, Z.G., Liu, Q.S., Liu, R., Hu, W., Deng, X.Y.: Influence of Rayleigh-Taylor instability on liquid propellant reorientation in a low-gravity environment. Chin. Phys. Lett. 26, 114701 (2009)

Marchetta, J.G., Roos, K.M.: Simulating magnetic positive positioning of cryogenic propellants in a transient acceleration field. Comput. Fluids 38, 843-850 (2009)

Scardovelli, R., Zaleski, S.: Direct numerical simulation of freesurface and interfacial flows. Annu. Rev. Fluid. Mech. 31, 567603 (1999)

Stark, J.A., Bradshow, R.D., Blatt, M.H.: Low-g Fluid Behavior Technology Summaries (1974). NASA-CR-134746 